\title{
Análisis cualitativo sobre las percepciones de extensionistas públicos salvadoreños en relación al maíz criollo
}

\author{
Análise qualitativa sobre as percepções dos extensionistas públicos \\ salvadorenhos em relação ao milho crioulo
Qualitative analysis on the perceptions of Salvadorian public extensionists related to creole maize

\author{
Carlos Ayala Durán ${ }^{1}$ \\ Paulo Dabdab Waquil ${ }^{2}$
}

Recebido em 21/09/2018; revisado e aprovado em 30/04/2019; aceito em 06/05/2019

DOI: http://dx.doi.org/10.20435/ inter.v20i3.2165

\begin{abstract}
Resumen: Este trabajo tiene como objetivo analizar las percepciones de extensionistas públicos salvadoreños $(n=39)$ en relación a la producción de maíz con semilla criolla. Para tal fin, se utiliza los sistemas agroalimentarios localizados como marco teórico, centrándose en su dimensión institucional. De esa forma, se emplearon cuestionarios semi abiertos en primer lugar y posteriormente una escala Likert para explorar las percepciones de los extensionistas sobre las diferentes semillas de maíz. Los resultados muestran características particulares de los extensionistas salvadoreños, como amplia experiencia profesional y una escasa formación a nivel de postgrado. En relación a sus percepciones, se evidencia una posición ambivalente, ya que se considera al maíz criollo como menos productivo que el maíz híbrido, si bien la semilla originaria se le asocian características agronómicas deseables como resistencia a plagas y sequías.
\end{abstract}

Palabras clave: El Salvador; maíz; sistemas agroalimentarios localizados.

Resumo: O presente trabalho tem como objetivo analisar as percepções de extensionistas públicos salvadorenhos ( $n=39)$ em relação à produção de milho com semente crioula. Para atingir esse objetivo, se utiliza os sistemas agroalimentares localizados como marco teórico, focando-se na sua dimensão institucional. Assim, foram utilizados questionários semi abertos e uma escala Likert para conhecer as percepções dos profissionais salvadorenhos. Os resultados mostram características particulares dos extensionistas, como a amplia experiência profissional e uma baixa formação em nível de pós-graduação. Em relação com as suas percepções, evidencia-se uma posição ambivalente, dado que se considera a semente crioula como menos produtiva que a híbrida, porém a semente nativa é associada a características agronómicas positivas, como resistência às pragas e à seca.

Palavras-chave: El Salvador; milho; sistemas agroalimentares localizados.

Abstract: The following work has as objective to analyze perceptions of Salvadorian rural extensionist's ( $n=39$ ) in relation to maize production using creole seed. To that end, Localized Agrifood Systems are employed as framework, particularly its institutional dimension. Thereof, semi open questionnaires and a Likert scale were employed to explore their views on maize seeds. Results show particular characteristics among extensionists, as their ample job experience and scarcity of postgraduate studies. Regarding their perceptions, this text shows and ambivalent position, given creole seed is seen as less productive than hybrid, although it is associated with desirable agronomic characteristics such as resistance to plagues and droughts.

Keywords: El Salvador; maize; localized agrifood systems.

\section{INTRODUCCIÓN}

Recientemente, se evidencia cómo a nivel mundial la opinión pública ha mostrado un creciente interés sobre el bienestar ambiental y de los agroecosistemas (WHEELER 2008a). Este interés genera de forma paralela un aumento en los esfuerzos de regulación agrícola así como un incremento en iniciativas que apoyan a agricultores para que adopten prácticas agrícolas más

\footnotetext{
${ }^{1}$ Pontifícia Universidade Católica do Rio Grande do Sul (PUC-RS), Porto Alegre, Rio Grande do Sul, Brasil.

${ }^{2}$ Universidade Federal do Rio Grande do Sul (UFRGS), Porto Alegre, Rio Grande do Sul, Brasil.
} 
sustentables (CHANG; KRISTIANSEN, 2006). Esta creciente regulación así como la adopción de técnicas sustentables puede suponer un reto adicional para aquellos países latinoamericanos con una alta participación del sector agrícola en sus respectivas economías. En ese contexto, y para un pequeño país como El Salvador, donde la cultura del maíz ha tenido una importancia histórica desde tiempos precolombinos (MINISTERIO DE EDUCACIÓN [MINEC], 2009; AYALA DURÁN; MIGUEL, 2016), prácticas amigables con el medio ambiente incluyen la agricultura orgánica y la producción de maíz utilizando semilla criolla. Sobre este último, la utilización de semilla criolla en la cultura de maíz tiene asociado unos requerimientos nutricionales menores, es más respetuosa con la biodiversidad y es consecuente con las formas de vida de los campesinos en cuestión.

Pese a ello, como producto del incremento en el uso de tecnologías asociadas a la revolución verde desde la segunda mitad del siglo pasado, la cultura del maíz en El Salvador ha visto sus prácticas sociotécnicas claramente modificadas, reduciéndose así la importancia del maíz criollo en la producción nacional de ese cereal. De tal forma, se ha potenciado tanto desde la arena pública como la privada, la paulatina substitución de semillas criollas originarias, por semillas mejoradas, destacadamente las semillas de origen híbrido. En ese sentido, el último censo agropecuario refleja que en todo el país, el 79.05\% del total del área plantada con maíz fue realizada con semilla híbrida (MINISTERIO DE ECONOMÍA [MINEC], 2009). Este nivel de penetración en el uso de semillas híbridas contrasta con otros países que poseen larga tradición en el cultivo del maíz, siendo que por ejemplo México obtiene $75 \%$ de su producción de maíz con semilla criolla, reduciéndose únicamente al $25 \%$ la producción con semilla híbrida (TADEOROBLEDO et al., 2015).

Simultáneamente, esta mudanza en la utilización de semillas híbridas en El Salvador tendrá aparejado unos altos costos de producción. De esa forma, Martínez (2007) indica que en la cultura de maíz en El Salvador, el costo de la adquisición de agroquímicos y semilla asciende al $33.7 \%$ y $4.6 \%$ respectivamente del total. De esa forma, casi el $40 \%$ del costo para producir maíz en El Salvador es atribuible únicamente a estos dos insumos.

El aumento en la utilización de semilla híbrida en El Salvador ha venido catalizado por el notable apoyo público al uso de este tipo de tecnología. Uno de los primeros pasos fue la legalización de estas prácticas en la década de los años setenta, mediante la promulgación de la Ley de Certificación de Semillas y Plantas (1971) seguida de Ley sobre Control de Pesticidas, Fertilizantes y Productos para Uso Agropecuario (1973). Paralelamente, desde el Centro Nacional de Tecnología Agropecuaria y Forestal (CENTA), órgano público especializado en investigación y extensión rural, se ha promovido históricamente la investigación, desarrollo, validación, transferencia y extensión de semillas híbridas de maíz a nivel nacional. Así por ejemplo, el mencionado centro público realiza actualmente la producción de progenitores del maíz híbrido H-59, para ser provisto a organizaciones privadas con fines comerciales, ya que estos últimos entes son los actores exclusivos encargados de la comercialización de los diferentes tipos de maíz a nivel nacional ${ }^{3}$. Similarmente, la semilla H-59 es la semilla más utilizada en el programa gubernamental de apoyo a la producción de granos básicos en todo el país.

\footnotetext{
${ }^{3}$ El Estado no vende directamente semilla a particulares. De tal suerte, se da la contradicción que CENTA produce los progenitores de maíz H-59, lo vende a entes privados para su reproducción y comercialización. Paralelamente, para la implementación de programas de apoyo al cultivo de granos básicos, el Estado se ve obligado a comprar insumos de actores privados, mismos que ayudó a desarrollar.
} 
Pese a que existe una notada predominancia en la utilización de técnicas oriundas de la revolución verde en la agricultura salvadoreña en general, y en la cultura del maíz en particular, comienzan a hacerse cada vez más patentes iniciativas públicas y privadas que promueven un rescate de los materiales genéticos criollos de maíz. En tal sentido, dentro del CENTA existe el Banco de Germoplasma, que colecta y almacena materiales genéticos criollos de una variedad de cultivos, incluyendo maíz. Asimismo, la agencia de extensión CENTA desarrolló e implementa actualmente un proyecto de biofertilizantes para el cultivo de granos básicos (CENTA, 2015). Paralelamente, desde el sector privado y organismos intragubernamentales, se han implementado Programas de Rescate de Variedades de Maíz Criollo (MORENO, 2000) o la elaboración de estudios sobre como producción y conservación de semillas criollas (ASOCIACIÓN FUNDACIÓN PARA LA COOPERACIÓN Y EL DESARROLLO COMUNAL DE EL SALVADOR [CORDES], 2016; RIVAS et al., 2013).

Si bien existen cada vez más iniciativas a nivel nacional y regional que buscan fomentar la producción de maíz con semilla criolla, parece existir una brecha en la información técnica sobre la utilización de este tipo de tecnología en el país, particularmente a nivel de agencias de extensión pública. En ese sentido, autores como Feder, Biner y Anderson (2011) resaltan que la reforma en muchos programas de extensión en países en desarrollo pasan necesariamente por la mejora en el acceso a la información de los agricultores. Siendo que la mayoría de la extensión en el mundo se realiza por medio de agencias públicas (ANDERSON; FEDER, 2007), estudios sobre extensionismo oficial cobrarían especial importancia en países como El Salvador y otros de América Latina, que poseen un sector agrícola amplio. Pese a ello, en Latinoamérica la investigación en temas de extensión rural no ha sido el foco de los estudios socio agrarios (MÉNDEZ, 2006), existiendo una particular carencia en relación a las visiones de los extensionistas rurales, profesionales que poseen contacto directo con los agricultores.

Aunque se reconozca esta relativa carencia, es posible identificar investigaciones recientes que estudian a los órganos públicos de extensión rural y sus extensionistas en América Latina. En el sur de Brasil por ejemplo, trabajos como los de Zilli, Bregt y Rossiter (2006) o Hötzel y Sneddon (2013) mostraban resultados pertinentes. Los primeros investigaron las percepciones de riesgo de extensionistas rurales y agricultores, en cuanto los segundos se centraron en el papel de los extensionistas rurales en la aplicación de prácticas de alivio del dolor al descornar novillos. En el vecino país Uruguay, Virginia (2016) se centró en el liderazgo de los extensionistas rurales, así como en la importancia de esta aptitud para la ejecución de sus labores. Más recientemente, diversos trabajos realizados por Fernando Landini han investigado a los extensionistas rurales de diversos países latinoamericanos como Uruguay (LANDINI; RIET, 2015), Brasil (LANDINI, 2015a), Ecuador (LANDINI; BIANQUI, 2014b) Paraguay (LANDINI, 2012; LANDIDI; BIANQUI; CRESPI, 2013) Argentina (LANDINI, 2016b; 2016d), El Salvador (LANDINI, 2015b); así como Latinoamérica en su conjunto (LANDINI; BIANQUI, 2014a; LANDINI, 2016a; 2016c).

El creciente interés en este tipo de investigaciones sobre extensión rural, y particularmente aquellas que abordan las visiones sobre los extensionistas rurales, parece no haber permeado plenamente en El Salvador. De esa forma, fue posible identificar tan solo un estudio que aborda exclusivamente las percepciones de extensionistas rurales en ese país (LANDINI, 2015b). En el mencionado trabajo, Landini (2015b) se centraba en los problemas enfrentados por extensionistas públicos salvadoreños, queriendo a su vez indagar cuál era su concepción en la extensión rural. Únicamente de forma tangencial, los extensionistas salvadoreños son sujeto de investigación en estudios como los de Ardila (2010), Escobar (2012), Landini y Bianqui (2014a) y Landini (2016a, 
2016c). En ese sentido, no fue posible identificar mayores investigaciones que se centrasen exclusivamente sobre extensionistas rurales salvadoreños, ni trabajos específicos que abordasen la visión de estos en relación a la cultura del maíz criollo/híbrido.

De esta forma, en virtud que las agencias públicas responsables por temas agrícolas y su personal son una importante fuente de información para los agricultores (ANDERSON; FEDER, 2007), a la importancia fundamental que reviste el maíz (y la tortilla de maíz) en la ingesta diaria de los salvadoreños (MENCHÚ; MÉNDEZ, 2011; AYALA, 2018), a que existe un porcentaje bajo en la utilización de semilla criolla en la producción nacional de maíz y a una relativa falta de investigaciones que tomen en consideración a los extensionistas rurales salvadoreños; el presente trabajo tiene como objetivo analizar la visión de extensionistas públicos rurales en El Salvador específicamente en lo que concierne la producción de maíz con semilla criolla. Paralelamente, este trabajo se muestra necesario para comenzar a entender la visión de estos funcionarios públicos, buscando sentar bases para descifrar su influencia en la alta utilización de semilla híbrida para en la producción nacional. Para tal fin, a continuación se definen cuáles fueron los materiales y métodos utilizados. Posteriormente se destacan los resultados y elabora su discusión. Finalmente, se abordan las consideraciones finales.

\section{MATERIALES Y MÉTODOS}

El presente trabajo forma parte de una investigación mayor, correspondiente a una parte de la tesis doctoral del primer autor. En ella, se combinan metodologías cuantitativas y cualitativas para predecir los factores que influyen en la recomendación para el uso de determinadas prácticas agrícolas por parte de los extensionistas rurales. El caso específico del presente artículo, es de naturaleza cualitativa, exploratoria y descriptiva. De esta forma, el presente trabajo tiene como marco teórico general los sistemas agroalimentarios localizados (SIAL). Los mismos fueron originalmente definidos como "Organizaciones de producción y servicios (unidades de producción agrícola, empresas agroalimentarias, comerciales, de servicio, gastronómicas...) asociadas por sus características y funcionamiento a un territorio específico" (CENTRE DE COOPERATION INTERNATIONALE EN RECHERCHE AGRONOMIQUE POUR LE DEVELOPPEMENT [CIRAD], 1996, p. 5). Siguiendo los aportes de Muchnik (2006), los sistemas agroalimentarios localizados estarían compuestos por cuatro dimensiones: Histórica, técnica, institucional y alimenticia. Específicamente la dimensión institucional, tendría como foco las relaciones entre los actores sociales y sus estrategias individuales y/o colectivas (MUCHNIK, 2006). Esta dimensión abarca arreglos entre productores, formación de cooperativas, sistemas de compras, actuaciones de los poderes públicos, entre otras. Como se ha mencionado, el alcance de los órganos públicos en relación a la agricultura convencional en general y a la producción del maíz en particular en El Salvador es amplio, abarcando esferas tan diversas como la provisión de créditos productivos, la investigación y desarrollo de semillas híbridas de cereales, la donación de paquetes agrícolas con semilla híbrida, la asesoría por parte de los servicios de extensión, entre otros. De esa forma, entre todos los arreglos institucionales (públicos y privados) circundantes a la producción de maíz en El Salvador, el siguiente artículo se centra en una parte de la dimensión institucional del SIAL, y busca analizar el papel de uno de los actores públicos más visibles y menos estudiados en el campo salvadoreño: Los extensionistas públicos rurales.

De esa forma, los sistemas agroalimentarios localizados, marco teórico del presente artículo, ya han sido utilizado en América Latina para el abordaje de diversos alimentos con un 
fuerte anclaje territorial como los quesos en Perú (BOUCHER, 2004) y Brasil (AMBROSINI; FILIPPI; MIGUEL, 2009), fresas (SPECHT, 2009) u hongos tipo cuatlicoche (SALCIDO et al., 2015). Dada la larga historia que reviste el maíz en El Salvador (MINED, 2009; AYALA DURÁN; MIGUEL, 2016) y en virtud que ese cereal presenta un anclaje territorial evidente en el país, se consideró que este marco teórico provee los instrumentos analíticos necesarios para poder realizar un estudio apropiado. De tal suerte, se considera que conocer la opinión de estos servidores públicos sobre la utilización de semilla híbrida, y los factores determinantes a la hora de recomendar su utilización, resulta fundamental para pensar en estrategias a futuro que sean más acordes con las necesidades locales de los agricultores. En ese sentido, Wheeler (2008b) manifiesta que el tener acceso a información fidedigna y relevante representa un componente crítico para influenciar a que los agricultores decidan adoptar prácticas alternativas, nuevas tecnologías e innovaciones. De esa forma, conocer la visión de estos extensionistas sobre maíz criollo, siendo que ellos son uno de los primeros actores públicos con los cuales los productores de maíz tienen contacto en sus respectivas parcelas, resulta fundamental.

Para tal fin, el presente artículo presenta un análisis exploratorio en relación a las percepciones sobre el maíz criollo de extensionistas públicos rurales provenientes de dos instituciones: La Escuela Nacional de Agricultura (ENA) y del Centro Nacional de Tecnología Agrícola y Forestal (CENTA). De esa forma, el presente estudio ha tomado en consideración un total de 39 extensionistas públicos, según lo muestra la tabla 1.

Tabla 1- Procedencia de los entrevistados

\begin{tabular}{l|c|l}
\hline \multicolumn{1}{c|}{$\begin{array}{c}\text { Procedencia de } \\
\text { extensionistas }\end{array}$} & $\begin{array}{c}\text { No. de } \\
\text { extensionistas }\end{array}$ & \multicolumn{1}{c}{ Observaciones } \\
\hline $\begin{array}{l}\text { Agencias de extensión del } \\
\text { departamento La Libertad }\end{array}$ & 20 & $\begin{array}{l}\text { Procedente de todas las agencias del departamento, a } \\
\text { saber: Atiocoyo, Quezaltepeque, Puerto de la Libertad, } \\
\text { San Juán Opico y Zapotitán }\end{array}$ \\
\hline $\begin{array}{l}\text { Agencias de extensión del } \\
\text { departamento Sonsonate }\end{array}$ & 8 & $\begin{array}{l}\text { Procedente de todas las agencias del departamento, a } \\
\text { saber: Armenia, El Peñón, Sonsonate }\end{array}$ \\
\hline $\begin{array}{l}\text { Escuela Nacional de } \\
\text { Agricultura }\end{array}$ & 3 & $\begin{array}{l}\text { Docentes de las diversas cátedras del técnico en } \\
\text { agronomía. }\end{array}$ \\
\hline $\begin{array}{l}\text { Centro Nacional de } \\
\text { Tecnología Agropecuaria y } \\
\text { Forestal }\end{array}$ & 8 & $\begin{array}{l}\text { Profesionales de los departamentos: Tecnología de } \\
\text { semillas, validación de semillas, socio economía, banco } \\
\text { de germoplasma. }\end{array}$ \\
\hline TOTAL & 39 & \\
\hline \hline
\end{tabular}

Fuente: Elaboración propia (2018).

El trabajo de campo se realizó en dos instancias. En primer lugar, se realizaron cuestionarios semi abiertos que fueron respondidos individualmente por todos los extensionistas públicos consultados. Estos cuestionarios iniciaban con preguntas de información demográfica para caracterizar a los extensionistas. Posteriormente, se indagaba la percepción de sobre la cultura de maíz utilizando semilla criolla/híbrida mediante preguntas semi abiertas. En esta etapa se aprovechó para indagar sobre el impulso gubernamental a la producción de semillas híbridas. Posteriormente, la visión de estos extensionistas fue explorada utilizando una escala Likert (15) con diversas afirmaciones. Las mismas eran basadas en estudios previos sobre maíz criollo (CORTEZ 2014; MADGALENO-HERNÁNDEZ et al., 2016) así como en posibles motivos que los 
extensionistas pueden concebir como decisivos en su evaluación de este tipo de tecnología. Se decidió realizar las preguntas abiertas en primer lugar y después utilizar la escala Likert con el objetivo que las afirmaciones contenidas en la mencionada escala no influenciaran las respuestas abiertas. En básicamente todos los casos, los cuestionarios fueron respondidos de forma presencial en la propia oficina del funcionario público, con la única excepción de los respondientes de una agencia en La Libertad (agencia Quezaltepeque), que enviaron el cuestionario vía e-mail. Posterior al llenado de los cuestionarios, se realizaban reuniones conjuntas con grupos de entre 3 - 5 extensionistas donde se discutía cuestiones relativas a su conocimiento sobre producción maíz criollo, con un doble fin: Obtener información cualitativa que pudiese no reflejarse en el cuestionario y obtener retroalimentación de las opiniones expresadas en ese instrumento. Este trabajo de campo se realizó entre los meses de Marzo 2017 hasta Febrero de 2018.

\section{RESULTADOS Y DISCUSIÓN}

Los extensionistas rurales sujetos a este estudio tienen una edad media de 50.08 años ( $\sigma=10.69)$, con 22.38 años en media de experiencia laboral ( $\sigma=10.24)$. La gran mayoría de ellos son hombres, habiendo únicamente entre sus filas cinco mujeres, lo que implicaría alrededor de un $12 \%$ de los extensionistas públicos correspondientes al sexo femenino. El estudio realizado por Landini y Bianqui (2014a) retratan leves diferencias a los extensionistas públicos salvadoreños sujetos de aquel estudio, siendo que en el caso de aquellos autores $(n=34)$, la media de edad en los extensionistas salvadoreños estudiados era 41.3 años, contando únicamente con 13.9 años de experiencia laboral. Asimismo, los 23.38 años de experiencia encontrados en los extensionistas del presente estudio son notablemente mayores a la media reportada por Landini y Bianqui (2014a) para extensionistas latinoamericanos ( $\sigma=11.1$ años).

Por otra parte, con la excepción de dos respondientes, todos los extensionistas del presente estudio tienen formación a nivel superior en temas agropecuarios, ya sea a nivel universitario o técnico (no universitario). Comparada esta información con los datos reportados para Latinoamérica por Landini y Bianqui (2014a), los extensionistas salvadoreños tienen relativamente un bajo nivel de formación, ya que únicamente un respondiente posee estudios a nivel de maestría. Entre los que poseen grado universitario completo, el $61.53 \%$ de los casos totales son formados en ingeniería agrícola $(n=24)$. De esa forma, es destacable la ausencia de profesionales de otras áreas comunes en los cuerpos extensionistas como zootecnia, veterinaria o ciencias sociales; identificadas por Landini y Bianqui (2014a) para América Latina. Adicionalmente, la mayoría de los extensionistas públicos rurales de estos dos departamentos proceden en su gran mayoría (84.6\%) de una familia con vocación agropecuaria, siendo que familiares se dedicaban a esta actividad.

Al ser preguntados sobre cuál es la principal fuente de información en temas agropecuarios, el $51.3 \%$ de ellos respondió que eran los diferentes documentos (investigaciones, manuales, capacitaciones, etc.) emanados de la agencia oficial de extensión rural: CENTA ( $n=20)$. Un 15.4\% respondieron que eran los colegas extensionistas $(n=6)$. Seguido en orden de importancia, un $12.8 \%$ citaba otras fuentes, $7.7 \%$ citaban al propio agricultor e igual porcentaje a investigaciones científicas $(n=3)$. Por otra parte, el restante $5.1 \%$ cita a información oficial del Estado como publicaciones, estadísticas, manuales, etc. En este sentido, una pequeña minoría señaló como principal fuente de información en temas agropecuarios a investigaciones científicas, ensayos de 
campo o revistas especializadas en la materia. Esto se puede deber parcialmente al relativamente bajo nivel de formación de los extensionistas, mencionado en el párrafo anterior. De particular importancia es el hecho que un $7.7 \%$ de los extensionistas citan al propio agricultor al cuál ellos están asesorando como la principal fuente de información. Sin desmeritar el conocimiento adquirido por los agricultores a lo largo del tiempo, la ausencia de una base técnica sólida por parte de los extensionistas rurales puede perjudicar la efectividad de la asesoría técnica que ellos proveen. En ese sentido, la investigación doctoral de la cuál surge el presente artículo da cuenta de conflictos existentes entre extensionistas y agricultores al haber tenido asesoría que resultó en la pérdida parcial de cosechas.

Posterior a esta información demográfica, se indaga las percepciones de los extensionistas en relación a la cultura de maíz utilizando semilla híbrida y criolla. Como se mencionó anteriormente, la alta utilización de semilla híbrida puede ser explicada parcialmente gracias al largo brazo del apoyo público a este tipo de tecnología. De tal suerte, desde los años dos mil el gobierno ejecutivo dona directamente a pequeños agricultores paquetes agrícolas conteniendo semilla de maíz y frijol, como forma de promover el cultivo de granos básicos en el país. Los beneficiarios del paquete de apoyo al cultivo de maíz reciben 25 libras (12.12 Kg) de semilla de maíz H-59, y 100 libras ( $45.45 \mathrm{Kg}$ ) del abono sulfato de amonio. En tal sentido, se preguntó a los extensionistas el por qué los paquetes gubernamentales cuentan fundamentalmente con semilla de maíz híbrida. El 61.5\% considera que el potencial de rendimiento de ese tipo de maíz es superior a otros, el $10.3 \%$ considera que es debido a mayor disponibilidad de semilla $(n=4)$; el $7.7 \%$ valora al potencial de rendimiento conjuntamente con la capacidad de esta ser producida por pequeños agricultores, mientras que el $20.5 \%$ restante citó otros motivos $(n=8)$. Las respuestas de los extensionistas ya evidencian la importancia del potencial de rendimiento y la disponibilidad de la semilla híbrida. Sobre este último, la investigación doctoral de la cuál se desprende este artículo ha podido certificar que en el departamento La Libertad (sede del Centro Nacional de Tecnología Agropecuaria y Forestal y de la Escuela Nacional de Agricultura), los agroservicios solamente ofrecen a la venta semilla de maíz híbrida. De forma paralela, el Estado produce progenitores únicamente para maíz híbrido, sin que exista desarrollo e investigación para maíz criollo. Esto levanta un serio inconveniente a la hora de querer valorar alternativas ambientalmente más limpias, como el cultivo de maíz utilizando semilla criolla, ya que existiría a priori una baja disponibilidad de este insumo. La escasez de semilla criolla es identificada también por Moreno (2000) en Chalatenango, departamento vecino de La Libertad.

Pese a este gran impulso gubernamental a la semilla de maíz híbrida, existe dentro de CENTA un Banco de Germoplasma que recolecta, cataloga y almacena material genético de una serie de cultivos, incluyendo maíz. Ante la pregunta por qué motivo existe este Banco que guarda semillas de maíz criollo, el 38.5\% de los extensionistas consideran que es importante para poder preservar el material genético $(n=15)$. Por su parte, el $25.6 \%$ de los respondientes ven un motivo más utilitario: el resguardo de material genético básico para el fitomejoramiento $(n=10)$. Conservar las variedades nativas de maíz cuenta con el $10.3 \%$ de las opiniones $(n=4)$. La utilización de esta semilla para que agricultores cultiven es citado en $5.1 \%$ de los casos $(n=2)$, mientras que $20.5 \%$ de las respuestas adujeron otros motivos $(n=8)$. De esta forma, solamente un pequeño número de respondientes ven útil el preservar la variedad de maíz criollo propio de El Salvador.

Por otra parte, se indagó cuál es la semilla de maíz que recomiendan estos extensionistas a los agricultores asesorados. En una buena parte de los casos, los respondientes expresan una 
diferencia a la hora de recomendar un tipo de semilla dependiendo de dos condicionantes: la finalidad y las posibilidades económicas del agricultor. En términos generales, cuando el agricultor tiene medios económicos necesarios, los extensionistas recomiendan semilla híbrida que suele ser más cara y tiene requerimientos nutricionales mayores. Por otra parte, cuando el agricultor tiene menor disponibilidad económica, se recomienda una semilla criolla. Los resultados generales los muestra la tabla 2.

Tabla 2- Maíz recomendado para que utilicen los agricultores

\begin{tabular}{|c|c|c|c|c|c|c|}
\hline & \multicolumn{3}{|c|}{$\begin{array}{l}\text { ¿Cuál maíz híbrido recomienda cultivar a } \\
\text { los agricultores con que usted trabaja? }\end{array}$} & \multicolumn{3}{|c|}{$\begin{array}{l}\text { ¿Cuál maíz criollo recomienda cultivar a } \\
\text { los agricultores con que usted trabaja? }\end{array}$} \\
\hline & & Frecuencia & $\%$ Valido & & Frecuencia & $\%$ Valido \\
\hline \multirow{5}{*}{ Válidos } & H59 & 13 & 43.3 & Santa Rosa & 7 & 36.84 \\
\hline & $\begin{array}{l}\text { H59 junto con otro } \\
\text { híbrido }\end{array}$ & 8 & 26.6 & Capulín & 4 & 21.05 \\
\hline & Otro & 9 & 30 & Otro & 8 & 42.10 \\
\hline & $\begin{array}{l}\text { No recomienda } \\
\text { cultivar maíz } \\
\text { híbrido }\end{array}$ & 8 & & $\begin{array}{l}\text { No recomienda } \\
\text { cultivar maíz } \\
\text { criollo }\end{array}$ & 19 & \\
\hline & Total & 38 & & - & 38 & \\
\hline Perdidos & $\begin{array}{l}\text { No sabe/No } \\
\text { responde }\end{array}$ & 1 & & $\begin{array}{l}\text { No sabe/No } \\
\text { responde }\end{array}$ & 1 & \\
\hline Total & & 39 & & & & \\
\hline
\end{tabular}

Fuente: Elaboración propia (2018).

Como se evidencia, el maíz H-59, parcialmente subsidiado por el Estado, es el principal maíz recomendado por los extensionistas, ya sea en solitario (43.3\%) o junto a algún otro híbrido (26.6\%). De tal forma, en casi el 70\% de los casos (válidos) este tipo de maíz es recomendado. El motivo más citado para recomendar maíz híbrido en general es el alto potencial de rendimiento, mismo que equivale al $33.3 \%$ de los casos. En orden de importancia decreciente, entre los motivos por los que se recomienda este tipo de semilla es: resistente a plagas/sequías (12.8\%), buena adaptación (10.3\%), semilla producida nacionalmente (10.3\%), requiere menos inversión (5.1\%) y otros motivos (5.1\%).

Por otra parte, el maíz criollo más recomendado es el Santa Rosa (36.4\%) seguido del capulín (21.05\%). Otros maíces criollos son citados en el $42.1 \%$ de los casos válidos. Entre los motivos que pesan a la hora de recomendar esa tecnología se encuentran la necesidad de menos inversión en dinero (7.7\%) y que es más resistente a sequías (7.7\%).

Una vez indagados estos aspectos mediante preguntas semi abiertas, se buscó conocer las percepciones de los extensionistas sobre semilla de maíz criolla, particularmente comparándola con la semilla híbrida mediante una escala Likert. El resumen de sus valoraciones están condensadas en la tabla 3. Las afirmaciones expuestas para valoración de los extensionistas se centraban en una variedad de características tanto de la semilla de maíz híbrida como criolla, atendiendo a aspectos productivos, adaptativos, nutritivos, económicos y cognitivos. 
Tabla 3- Escala Likert (1-5) en relación a valoración de semilla criolla e híbrida

\begin{tabular}{|c|c|c|c|c|c|}
\hline & $\mathrm{N}$ & Min. & Max. & Media & Desv. Std. \\
\hline El maíz híbrido tiene mejores rendimientos que el maíz criollo* & 39 & 1 & 5 & 4.05 & 1.123 \\
\hline $\begin{array}{l}\text { El maíz híbrido produce mazorcas y granos de maíz más } \\
\text { uniformes (homogéneos) que el maíz criollo * }\end{array}$ & 39 & 1 & 5 & 3.59 & 1.229 \\
\hline $\begin{array}{l}\text { El maíz híbrido tiene mayor rendimiento para forraje (silo) que el } \\
\text { maíz criollo* }\end{array}$ & 39 & 1 & 5 & 3.23 & 1.287 \\
\hline $\begin{array}{l}\text { El maíz híbrido presenta mejor rendimiento de masa que el maíz } \\
\text { criollo * }\end{array}$ & 39 & 1 & 5 & 3.21 & 1.260 \\
\hline $\begin{array}{l}\text { El maíz híbrido es más resistente a sequías, en comparación el } \\
\text { maíz criollo + }\end{array}$ & 39 & 1 & 4 & 2.41 & 1.044 \\
\hline $\begin{array}{l}\text { El maíz híbrido es más resistente a plagas, en comparación el } \\
\text { maíz criollo + }\end{array}$ & 39 & 1 & 5 & 2.49 & 1.073 \\
\hline $\begin{array}{l}\text { El maíz híbrido está mejor adaptado a las condiciones climáticas } \\
\text { de EL SALVADOR que el maíz criollo + }\end{array}$ & 39 & 1 & 5 & 2.38 & 1.269 \\
\hline $\begin{array}{l}\text { El maíz híbrido presenta en términos generales mejores índices } \\
\text { en nutrientes como hierro y zinc que el maíz criollo / }\end{array}$ & 39 & 1 & 5 & 2.95 & 1.255 \\
\hline $\begin{array}{l}\text { El maíz híbrido presenta en términos generales una cantidad de } \\
\text { proteína superior al maíz criollo / }\end{array}$ & 39 & 1 & 5 & 2.59 & 1.208 \\
\hline $\begin{array}{l}\text { El maíz híbrido es preferido por los compradores o } \\
\text { intermediarios de maíz } \$\end{array}$ & 39 & 1 & 5 & 3.97 & 1.158 \\
\hline $\begin{array}{l}\text { El maíz híbrido es preferido por la población en general que } \\
\text { compra y consume maíz. \$ }\end{array}$ & 39 & 1 & 5 & 3.15 & 1.288 \\
\hline $\begin{array}{l}\text { El maíz híbrido es más deseado que el maíz criollo por parte de } \\
\text { personas que se dedican a vender / procesar comida (pupusas, } \\
\text { tamales, panaderías, etc.) } \$\end{array}$ & 39 & 1 & 5 & 3.33 & 1.305 \\
\hline $\begin{array}{l}\text { El maíz híbrido es mejor que el maíz criollo para la alimentación } \\
\text { animal \$ }\end{array}$ & 39 & 1 & 5 & 3.00 & 1.257 \\
\hline $\begin{array}{l}\text { La producción de maíz utilizando semilla híbrida es más cara que } \\
\text { utilizando semilla criolla } \$\end{array}$ & 39 & 1 & 5 & 4.49 & .854 \\
\hline $\begin{array}{l}\text { La producción de maíz utilizando semilla híbrida es } \\
\text { económicamente más lucrativo que utilizando semilla criolla \$ }\end{array}$ & 39 & 1 & 5 & 3.92 & 1.133 \\
\hline $\begin{array}{l}\text { Las personas que dan asesoría técnica sobre producción de maíz } \\
\text { conocen sobre la producción con semilla criolla \& }\end{array}$ & 39 & 1 & 5 & 3.10 & 1.165 \\
\hline $\begin{array}{l}\text { El maíz híbrido es más conocido que el maíz criollo por parte de } \\
\text { los agricultores \& }\end{array}$ & 39 & 1 & 5 & 3.36 & 1.158 \\
\hline $\begin{array}{l}\text { Durante mi formación en agricultura, era abordada la producción } \\
\text { de maíz con semilla criolla \& }\end{array}$ & 39 & 1 & 5 & 3.10 & 1.429 \\
\hline $\begin{array}{l}\text { Es importante que el Banco de Germoplasma almacene semillas } \\
\text { de maíz criollas originarias de El Salvador }\end{array}$ & 39 & 4 & 5 & 4.85 & . 366 \\
\hline Validos N (listwise) & 39 & & & & \\
\hline
\end{tabular}

Símbolos: *: Características productivas. +: Características de adaptación. /: Características nutricionales. \$: Características económicas. \&: Características cognitivas.

Fuente: Elaboración propia (2018).

En relación a aspectos productivos, el que es valorado más positivamente por parte de los extensionistas entrevistados es el potencial de rendimiento del maíz híbrido en relación al maíz criollo ( $\mu=4.05)$. Este suele ser el principal argumento, tanto a nivel público como privado, para la provisión de semilla híbrida de maíz. Este aspecto se ve seguido de la posibilidad del maíz criollo de producir mazorcas y granos más uniformes $(\mu=3.59)$ y un mejor rendimiento para silo 
( $\mu=3.23)$ y rendimiento en masa superior al maíz criollo $(\mu=3.21)$. Siendo que la mayoría del maíz en El Salvador es consumido en forma tortilla, el tema de la producción de masa para la elaboración de ese platillo se vuelve fundamental. Esta valoración sobre el rendimiento del maíz criollo para masa de tortilla contrasta con la opinión de población que consume maíz e incluso de las pequeñas unidades molineras de elaboración de masa, ya que la investigación doctoral de la que se desprende este artículo ha atestiguado que diversos actores expresan disconformidad con el desempeño de algunos tipos de maíz híbrido a la hora de elaborar tortillas. En tal sentido, personas dueñas u operarias de molino consideran que el grano del híbrido H-59 es más duro, lo que genera una necesidad de mantenimiento mecánico más regular a los molinos.

Por otra parte, y pese a que estas características productivas son deseadas en la cultura del maíz, los mismos extensionistas reconocen que el maíz híbrido no es más resistente a sequías $(\mu=2.41)$, plagas $(\mu=2.49)$ y que está menos adaptado a las condiciones climáticas del país ( $\mu=2.38$ ); comparado con el maíz criollo. De tal suerte, bajo la visión de estos extensionistas, pese a que el maíz híbrido tiene mayor potencial de rendimiento, implicaría que la producción tiene mayores riesgos asociados.

En relación a aspectos nutricionales, los extensionistas consideran que el maíz híbrido no tiene mejores índices de proteína que el criollo $(\mu=2.59)$, mientras que no existe una postura clara en relación a nutrientes como hierro y zinc $(\mu=2.95)$. Sobre este particular, es destacable que el mismo CENTA desarrolla, produce y valida semilla de maíz de alta calidad proteica, como es el caso del maíz híbrido Oro Blanco (CENTA, 2008), Platino (CENTA, 2008) o H-CAS (CENTA, 2016). Pese a ello, los extensionistas no ven en términos generales al maíz híbrido superior al criollo en términos proteicos. Por su parte, existen estudios emanados desde la Escuela Nacional de Agricultura que colocan a maíces criollos salvadoreños con índices similares e incluso superiores a maíces híbridos. De tal suerte Laboratorio de Química Agrícola (LQA, 2015) retrata que las variedades criollas Negrito, Taberón, Olote rosado, Sapo y Chalatenango poseen índices superiores de proteína que el híbrido comercial Dekalb 390. De la misma forma, todos los maíces criollos estudiados en ese trabajo presentaron mejores índices en contenido de hierro que el maíz híbrido Dekalb 390 (LQA, 2015).

En relación a aspectos económicos, existe un entendimiento que la producción con semilla híbrida es más costosa que con semilla criolla ( $\mu=4.49)$. Pese a ello, los extensionistas consideran en buena medida que los compradores o intermediarios agrícolas prefieren el maíz híbrido que su par criollo ( $\mu=3.97)$. Esto parece no encontrar total correspondencia con los datos recolectados, ya que la investigación doctoral a la cuál pertenece este artículo, retrató que intermediarios agrícolas en La Libertad no tienen preferencia a la hora de comprar (con fines comerciales) maíz. Los mismos expresaban que no acostumbran comprar maíz criollo debido a que no suele haber disponibilidad de este tipo de cereal en particular. Otros aspectos económicos en orden de importancia decreciente según la media obtenida son: La producción con semilla híbrida es más lucrativa $(\mu=3.92)$, las personas que procesan-venden comida prefieren maíz híbrido ( $\mu=3.33$ ) y el maíz híbrido es preferido por las personas que compran y consumen maíz ( $\mu=3.15$ ). Nuevamente, estas afirmaciones no encuentran total correspondencia con los datos colectados en la investigación doctoral, ya que consumidores y vendedores de maíz consultados no suelen preferir explícitamente maíz híbrido, siendo que en muchos de los casos, tanto consumidores como vendedores no conocen el tipo de semilla adquirida. En otras ocasiones, operadores de molino o vendedoras de tortilla han expresado que algunos híbridos como el H-59 producen 
masa y tortillas mucho más duras, lo que dificulta el procesamiento, consumo y digestión de alimentos que utilizan ese maíz en particular.

Finalmente, los aspectos cognitivos por los que los extensionistas valoran al maíz híbrido y criollo tienen medias similares. De esa forma, los extensionistas consideran que el maíz híbrido es más conocido que el criollo ( $\mu=3.36)$, su formación formal incluía producción de maíz usando semilla criolla $(\mu=3.10)$ y los extensionistas en general conocen sobre producción con semilla criolla ( $\mu=3.10)$. Con ello, aparentemente los extensionistas conocen sobre este tema, siendo que las barreras al uso de esta tecnología no serían de orden cognitivo.

\section{CONSIDERACIONES FINALES}

Dado el bajo nivel de investigación en temas de extensión rural en América Latina en general (MÉNDEZ, 2006) y en El Salvador en particular, el presente artículo se ha centrado en investigar la percepción de extensionistas rurales públicos en El Salvador en relación a la cultura de maíz utilizando semilla criolla, comparándola con semilla híbrida. Para tal fin, el presente trabajo ha centrado su atención en la dimensión institucional de los sistemas agroalimentarios localizados, según la propuesta de Muchnik (2006).

Por otra parte, los resultados muestran que las percepciones de los extensionistas rurales sobre la cultura de maíz utilizando semilla criolla e híbrida presentan valoraciones ambivalentes. De esa forma, los extensionistas consideran que la producción con semilla criolla posee menor potencial de rendimiento y de producir mazorcas con granos uniformes que el maíz híbrido; si bien reconocen que la semilla criolla es más barata y menos vulnerable a plagas. Adicionalmente, los extensionistas consideran que la semilla híbrida es más deseada por consumidores e intermediarios de maíz, pese a que la evidencia recogida en el trabajo de campo apunta en otra dirección. Adicionalmente, resulta destacable que parte de estas percepciones parecen no estar cimentados sobre una base técnica científica, en virtud de al menos dos motivos: a) No existen amplios estudios sobre producción de maíz con semilla criolla en el país y, b) la principal fuente de información en temas agropecuarios para el 53.1\% de los respondientes es la agencia pública CENTA, organismo que no realiza a priori investigación y desarrollo de maíz criollo, con la notable excepción del Banco de Germoplasma.

Finalmente, siendo que los extensionistas de este artículo toman en consideración aspectos productivos, económicos, adaptativos, cognitivos y nutricionales a la hora de recomendar la utilización de un tipo de semilla de maíz, se necesitan estudios más amplios que puedan determinar y cuantificar en qué medida estos aspectos en particular afectan la recomendación para el uso de maíz criollo, dada la patente importancia de las agencias públicas de extensión en países en desarrollo.

\section{REFERENCIAS}

AMBROSINI, Larissa; FILIPPI, Eduardo; MIGUEL, Lovois. Produção de queijo serrano: estratégia de reprodução social dos pecuaristas familiares do sul do Brasil sob a perspectiva muldisciplinar do sistema agroalimentar localizado - SIAL. Estudo \& Debate, Lajeado, RS, v. 16, n. 2, p. 27-54, 2009.

ANDERSON, Jock R.; FEDER, Gershon. Agricultural extension. In: EVENSON, R.; PINGALI, P. (Ed.). Handbook of agricultural economics. Ámsterdam: Elsevier, 2007. v. 3, p. 2343-78.

ARDILA, Jorge. Extensión rural para el desarrollo de la agricultura y la seguridad alimentaria: aspectos conceptuales, situación y una visión de futuro. San José, Costa Rica: Instituto Interamericano de Cooperación para la Agricultura, 2010. 
ASOCIACIÓN FUNDACIÓN PARA LA COOPERACIÓN Y EL DESARROLLO COMUNAL DE EL SALVADOR (CORDES). Técnicas de producción, conservación bancos de semillas criollas. San Salvador: CORDES/Centro de Estudios Rurales y Agricultura Internacional-Unión Europea, 2016.

AYALA DURÁN, Carlos; MIGUEL, Lovois. Acumulación de riqueza: sistemas agrarios en la región norte del departamento de La Libertad, El Salvador. Ágora, Santa Cruz do Sul, v. 18, n. 2, p. 134-49, jul./dic. 2016.

AYALA, Carlos. Sistema Agroalimentario localizado del maíz blanco en Ciudad Arce, municipio de El Salvador. 2018. Tesis (Doctorado en Desarrollo Rural) - Universidad Federal do Rio Grande do Sul, Porto Alegre, 2018.

BOUCHER, François. Enjeux et difficultes d'une strategie collective d'activation des concentrations d'agroindustries rurales, le cas des fromageries rurales de Cajamarca, au Perou. 2004. Tesis (Doctorado en Economia)- Université de Versailles, Saint-Quentin-en-Yvelines, Paris, 2004.

CENTRE DE COOPERATION INTERNATIONALE EN RECHERCHE AGRONOMIQUE POUR LE DEVELOPPEMENT (CIRAD). Systèmes agroalimentaires localises: organisations, innovations et developpement local orientations et perspectives issues de la consultation du CIRAD. Paris: CIRAD-SAR, 1996.

CENTRO NACIONAL DE TECNOLOGÍA AGROPECUARIA Y FORESTAL (CENTA). CENTA H-CAS. Híbrido de maíz de alta calidad proteica para nutrir a El Salvador. San Andrés, La Libertad: CENTA, 2016.

CENTRO NACIONAL DE TECNOLOGÍA AGROPECUARIA Y FORESTAL (CENTA). Proyecto: Biofertilizantes en cultivo de maíz, frijol y café como alternativa agroecológica para una producción sostenible en El Salvador. San Andrés: CENTA, 2015.

CENTRO NACIONAL DE TECNOLOGÍA AGROPECUARIA Y FORESTAL (CENTA). Híbridos de maíz de alta calidad protéica Oro Blanco y Platino. Boletín Técnico 20. San Andrés, La Libertad: CENTA, 2008.

CHANG, Hui-Shung; KRISTIANSEN, Paul. Selling Australia as clean and green. Australian Journal of Agricultural \&Resource Economics, Adelaide, v. 50, n. 1, p. 103-13, mar. 2006.

CORTEZ, Manuel. Fitomejoramiento de maíces criollos utilizando la técnica de selección masal estratificada como apoyo al productor indígena salvadoreño. San Andrés, La Libertad: Escuela Nacional de Agricultura, 2014.

ESCOBAR, Germán. Diseño de una agenda de extensión rural latinoamericana para un desarrollo rural inclusivo. Extensión rural con un enfoque participativo y de mercado: hacia un marco conceptual. Santiago, Chile: Centro Latinoamericano para el Desarrollo Rural, 2012.

FEDER, Gershon; BIRNER, Regina; ANDERSON, Jock. The private sector's role inagricultural extension systems: potential and limitations. Journal of Agribusiness in Developing and Emerging Economies, Bingley, v. 1, n. 1, p. 31-54, 2011.

HÖTZEL, Maria; SNEDDON, Joanne. The role of extensionists in Santa Catarina, Brazil, in the adoptionand rejection of providing pain relief to calves for dehorning. Journal of Dairy Science, [s.I.], v. 96, n. 3, p. 1535-48, 2013.

LABORATORIO DE QUÍMICA AGRÍCOLA (LQA). Análisis bromatológico: porcentaje de proteína, promedio de hierro y zinc (partes por millón). San Andrés, La Libertad: Mirian Álvarez de Amaya (Escuela Nacional de Agricultura), 2015.

LANDINI, Fernando. Diversidad de expectativas de los extensionistas rurales latinoamericanos sobre la psicología. Estudos de Psicologia, [s.l.], v. 21, n. 4, p. 392-402, 2016. 
LANDINI, Fernando. Enfoques y prácticas de extensión rural públicas en el noreste argentino. Revista de Economia e Sociologia Rural, Brasília, v. 54, n. 1, p. 167-86, 2016 b.

LANDINI, Fernando. Problemas de la extensión rural en América Latina. Perfiles Latinoamericanos, Ciudad de México, v. 24, n. 47, p. 47-68, 2016c.

LANDINI, Fernando. Unfolding the knowledge and power dynamics of the 'farmers-rural extensionists' interface in North-Eastern Argentina. The Journal of Agricultural Education and Extension, [s.I.], v. 22, n. 5, p. 1-15, 2016d.

LANDINI, Fernando. Problemas enfrentados por extensionistas rurais brasileiros e sua relação com suas concepções extensão rural. Ciência Rural, [s.l.], v. 45, n. 2, p. 371-7, 2015a.

LANDINI, Fernando. Problemas percibidos y concepciones de extensión de los técnicos del Centro Nacional de Tecnología Agropecuaria y Forestal de El Salvador, Centroamérica. Interações, Campo Grande, MS, v. 16, n. 2, p. 455-64, jul./dic. 2015b.

LANDINI, Fernando. Problemas en la extensión rural paraguaya: modelos de extensión en la encrucijada. Cuadernos de Desarrollo Rural, Bogotá, v. 9, n. 69, p. 127-49, 2012.

LANDINI, Fernando; BIANQUI, Vanina. Socio-demographic profi le of different samples of Latin American rural extensionists. Ciência Rural, [s.l.], v. 44, n. 3, p. 575-81, 2014 a.

LANDINI, Fernando; BIANQUI, Vanina. La extensión rural en Ecuador desde el punto de vista de sus extensionistas. Revista de la Facultad de Agronomía, Zulia, v. 31, n. 3, p. 433-54, 2014b.

LANDINI, Fernando; BIANQUI, Vanina; CRESPI, Melina. Evaluación de las creencias sobre extensión rural de los extensionistas paraguayos. PSIENCIA. Revista Latinoamericana de Ciencia Psicológica, [s.I.], v. 5, n. 1, p. 3-14, 2013.

LANDINI, Fernando; RIET, Leonardo. Extensión rural en Uruguay: problemas y enfoques vistos por sus extensionistas. Mundo Agrario, La Plata, v. 16, n. 32, p. 1-17, 2015.

MAGDALENO-HERNÁNDEZ, Edgar et al. Selección tradicional de semilla de maíz criollo. Agricultura, sociedad y desarrollo, Ciudad México, v. 13, n. 3, p. 437-47, 2016.

MARTÍNEZ, Gerson. Competitividad agrícola en Centroamérica. Implicaciones de las normas de propiedad intelectual contenidas en el CAFTA-DR con relación al mercado de agroquímicos. San Salvador: FUNDE, 2007.

MENCHÚ, María; MÉNDEZ, Humberto. Análisis de la situación alimentaria en El Salvador, Guatemala: Instituto de Nutrición de Centroamérica y Panamá, 2011.

MÉNDEZ, Marlon. Los retos de la extensión ante una nueva y cambiante noción de lo rural. Revista Facultad Nacional de Agronomía, Medellín, v. 59, n. 2, p. 3407-23, 2006.

MINISTERIO DE EDUCACIÓN (MINED). Historia 2 de El Salvador. 2. ed. San Salvador: MINED, 2009.

MINISTERIO DE AGRICULTURA Y GANADERÍA (MAG). MAG beneficia a centenares de productores de hortalizas con casas malla. Noticias del Ministerio de Agricultura y Ganadería, 7 jul. 2016. Disponible en: https://tinyurl.com/y6ur5c4n.

MINISTERIO DE ECONOMÍA DE EL SALVADOR (MINEC). IV Censo Agropecuario 2007-2008. Resultados Nacionales. San Salvador, El Salvador, 2009. 
MORENO, Sandra. El maíz criollo se niega a desaparecer. El Diario de Hoy, 26 dic. 2000. Disponible en: https://tinyurl.com/ybnuw2x9.

MUCHNIK, José. Sistemas Agroalimentarios Localizados: evolución del concepto y diversidad de situaciones. In: CONGRESO INTERNACIONAL DE LA RED SIAL: Alimentación y Territorios, 3., 2006, Baeza - España. Anales [...]. Baeza, 2006.CD-ROM.

SALCIDO, Gerardo et al. Agricultura familiar y sistema agroalimentario localizado: Políticas locales para la producción de cuitlacoche (Ustílago Maydis sp.). Agricultura, Sociedad y Desarrollo, Ciudad de México, v. 12, n. 2, p. 199-218, 2015.

SPECHT, Suzymari. O território do morango no Vale do Caí: análise pela perspectiva dos sistemas agroalimentares localizados. 2009. Tesis (Doctorado en Desenvolvimento Rural) - Universidade Federal do Rio Grande do Sul, Porto Alegre, 2009.

RIVAS, Gonzalo et al. Bancos Comunitarios de Semillas Criollas: Una opción para la conservación de la agrobiodiversidad. Turrialba, Costa Rica: Centro Agronómico Tropical de Investigación y Enseñanza, 2013.

TADEO-ROBLEDO, Margarita et al. Rendimiento de maíces nativos e híbridos en diferente fecha de siembra y sus unidades calor. Revista Mexicana de Ciencias Agrícolas, Texcoco, v. 6, n. 1, p. 33-43, feb. 2015.

VIRGINIA, Gravina. Percepciones sobre el liderazgo de los extensionistas rurales en Uruguay, un abordaje cuantitativo. Agrociencia Uruguay, Montevideo, v. 20, n. 2, p. 145-52, 2016.

WHEELER, Sarah. Exploring professional attitudes towards organic farming, genetic engineering, agricultural sustainability and research issues in Australia. Journal of Organic Systems, [s.I.], v. 3, n. 1, p. 37-56, 2008a.

WHEELER, Sarah. What influences agricultural professionals' views towards organic agriculture? Ecological Economics, Ámsterdam, v. 65, n. 1, p. 145-54, 2008b.

ZILLI, Ivan; BREGT, Arnold; ROSSITER, David. A participatory approach for integrating risk assessment into rural decision-making: a case study in Santa Catarina, Brazil. Agricultural Systems, Ámsterdam, v. 87, n. 2, p. 229-44, 2006.

\section{Sobre los autores:}

Carlos Ayala Durán - Doctor en Desarrollo Rural- Universidade Federal do Rio Grande do Sul. Integrante del núcleo de Estudios en Economía Agraria de la Universidad Federal do Rio Grande do Sul. Enfocado en investigación pós doctoral en la Pontifícia Universidade Católica do Rio Grande do Sul (PUCRS). E-mail: carlos.ayala@ufrgs.br, Orcid: http://orcid.org/0000-0003-4049-671X

Paulo Dabdab Waquil - Doctor en Economía Agrícola - Universidad de Wisconsin (Madison). Profesor del Departamento de Ciências Econômicas e Relações Internacionais. Universidade Federal do Rio Grande do Sul. E-mail: waquil@ufrgs.br, Orcid: http://orcid.org/0000-0002-9430-7040 